\title{
Aspectos morfológicos e de incubação em três espécies de Corbicula Mühlfeld, no lago Guaíba, Rio Grande do Sul, Brasil (Bivalvia, Corbiculidae)
}

\author{
Demetrius da Silva Martins ${ }^{1}$, Inga Ludmila Veitenheimer-Mendes ${ }^{2} \&$ Maria Cristina Faccioni-Heuser $^{3}$
}

Biota Neotropica v6 (n2) -http://www.biotaneotropica.org.br/v6n2/pt/abstract?article+bn02806022006

\author{
Recebido em 15/09/05. \\ Versão reformulada recebida em 18/04/06 \\ Publicado 07/06/06
}
${ }^{1}$ Laboratório de Malacologia , Instituto de Biociências, UFRGS (www.ufrgs.br) , Av. Bento Gonçalves, 9500, prédio 43435, 91501-970 Porto Alegre, RS Bolsista Capes, autor para correspondência. (e-mail: demetriusmartins@yahoo.com.br)
${ }^{2}$ Laboratório de Malacologia, Instituto de Biociências, UFRGS (e-mail: inga.mendes@ufrgs.br)
${ }^{3}$ Centro de Microscopia Eletrônica, UFRGS (e-mail: mcfheuser@yahoo.com.br)

\begin{abstract}
Martins, D.S.; Veitenheimer-Mendes, I.L. and Faccioni-Heuser, M.C. Morphological aspects and incubation in three species of Corbicula Mühlfeld, in Guaíba Lake, Rio Grande do Sul, Brazil (Bivalvia, Corbiculidae).Biota Neotrop. Mai/ Aug 2006 vol. 6 no. 2, http://www.biotaneotropica.org.br/v6n2/pt/abstract?article+bn02806022006. ISSN 1676-0603

Three species of Corbicula identified in Guaíba Lake are compared in respect to the external morphology of their soft parts and aspects of branchial incubation. Morphological differences are most obvious in the siphons. For the first time, individuals of $C$. fluminea have been found with incubation occurring in the outer as well as the inner demibranchs. Individuals of $C$. largillierti showed incubation only in the inner demibranchs. In specimens identified as C. aff. fluminalis, tetrabranchial incubation predominated
\end{abstract}

Key words: Freshwater clams, siphons, viviparity, Corbicula fluminea, Corbicula largillierti, $\underline{\text { Corbicula. aff. fluminalis }}$

\section{Resumo}

Martins, D.S.; Veitenheimer-Mendes, I.L. and Faccioni-Heuser, M.C. Aspectos morfológicos e incubação em três espécies de Corbicula Mühlfeld, no lago Guaíba, Rio Grande do Sul, Brasil (Bivalvia, Corbiculidae).Biota Neotrop. Mai/Aug 2006 vol. 6 no. 2, http://www.biotaneotropica.org.br/v6n2/pt/abstract?article+bn02806022006. ISSN 1676-0603

Três espécies de Corbicula registradas para o lago Guaíba são comparadas quanto à morfologia externa das partes moles e aspectos relativos à incubação branquial. Observam-se diferenças específicas nos sifões e quanto à incubação branquial: espécimes de $C$. largillierti apresentam incubação somente nas demibrânquias internas; registra-se pela primeira vez espécimes de $C$. fluminea com incubação nas demibrânquias externas; e espécimes de $C$. af. fluminalis apresentam incubação predominantemente tetragenous (i. e. em ambas demibrânquias).

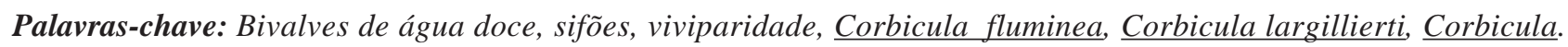
aff. fluminalis 


\section{Introdução}

A ampla distribuição geográfica e a capacidade de ocupação de vários tipos de habitat de água doce por Corbicula Mühlfeld, 1811 (McMahon 1983), desde sua introdução na América do Norte na década de 1920 (Counts 1986), são consideradas como os principais fatores responsáveis pelas variações na forma e coloração apresentadas nas conchas de espécimes dos diferentes táxons do gênero. Tais variações têm conduzido a inúmeras confusões taxonômicas em Corbicula, principalmente em virtude da maioria dos trabalhos estarem baseados apenas em caracteres da concha (Morton 1986, Araújo et al. 1993).

Britton \& Morton (1979), visando esclarecer esta confusão, apresentaram pela primeira vez diferenças em relação às partes moles para espécimes de Corbicula fluminea (Müller, 1774) e C. fluminalis (Müller, 1774), enfocando principalmente características do sifão exalante e diferenças reprodutivas. A maioria dos trabalhos se refere às características morfológicas de C. fluminea, em decorrência de sua importância e principalmente pelos grandes prejuízos causados na América do Norte, em especial, as questões relacionadas com a captação de água para fins de irrigação, industrial e geração de eletricidade (Britton \& Morton 1982, Araújo, Moreno \& Ramos 1993, Korniushin \& Glaubrecht 2002). Os autores Harada \& Nishino (1995) enfocaram aspectos do sifão inalante para diferenciação de espécies japonesas de Corbicula. No Brasil são poucos os estudos relativos a características morfológicas de Corbicula, Mansur \& Meier-Brook (2000) descreveram alguns aspectos da concha e partes moles de espécimes de $C$. fluminea oriundas do lago Guaíba em comparação com espécimes da família Sphaeriidae.

Os bivalves de água doce apresentam uma reprodução altamente especializada para exploração deste ambiente (Morton 1991). Enquanto a maioria dos bivalves marinhos é ovípara, muitos bivalves de água doce exibem várias formas de ovoviviparidade e viviparidade (Korniushin \& Glaubrecht 2003), como por exemplo as famílias da ordem Unionoida e duas famílias de Veneroida: Sphaeriidae e Corbiculidae. Park \& Ó Foighil (2000) comentam que as similaridades existentes entre Sphaeriidae e Corbiculidae - desenvolvimento de marsúpio, incubação das larvas e cuidado parental dos juvenis - se constituem em convergências adaptativas para ambientes de água doce.

Bivalves límnicos geralmente utilizam as brânquias para incubar seus embriões (Mackie 1986). Os corbiculídeos são típicos exemplos de bivalves que incubam seus embriões (Morton 1991). Segundo Ituarte (1984a) tal comportamento pode estar relacionado à proteção e desenvolvimento da prole como resposta às variações bióticas e abióticas dos ambientes límnicos.

Com relação às espécies de Corbiculidae há registros de um grande número de modos de desenvolvimento embrionário que varia desde a larva do tipo véliger livre natante, atribuído geralmente às espécies de águas salobras, como Corbicula cf. fluminalis (Müller, 1774), C. japonica Prime, 1864 e Polymesoda (Geloina) erosa (Solander, 1786) (Morton, 1982, 1985, 1986), até espécies que desenvolveram um cuidado parental dos juvenis com a redução ou completa ausência da larva pelágica (Park \& Ó Foighil 2000), como é o caso da espécie neotropical Cyanocyclas limosa (Maton, 1809) que incuba seus embriões até aproximadamente $1 / 4$ do tamanho da concha mãe (Parodiz \& Hennings 1965, Focht \& Veitenheimer-Mendes 2001).

A incubação em Corbiculidae é restrita aos táxons de água doce (Morton 1986), sendo melhor conhecida para $C$. fluminea (Müller, 1774) que se encontra distribuída em vários locais da América do Norte, América do Sul, África e Europa (Morton 1986, Kraemer \& Galloway 1986, Araújo et al. 1993, Ituarte 1994). Além dessa espécie, há registro de incubação para C. leana (Prime, 1864), C. australis (Lamarck, 1818), C. largillierti (Philippi, 1844), C. moltkiana Prime, 1878, C. matannensis Sarasin \& Sarasin, 1898, C. loehensis Kruimel, 1913, C. linduensis Bollinger, 1914, C. possoensis Sarasin \& Sarasin, 1898, Possostrea anomioides Bogan \& Bouchet, 1998 e Cy. limosa (Parodiz \& Hennings 1965, Ituarte 1984b, Morton 1986, Bogan \& Bouchet 1998, Byrne et al. 2000, Focht \& Veitenheimer-Mendes 2001, Korniushin \& Glaubrecht 2003).

Com exceção de C. possoensis que, segundo Korniushin \& Glaubrecht (2003), é a única espécie de Corbicula tipicamente tetragenous, isto é com incubação nas demibrânquias internas e externas, todas as demais espécies de Corbicula, estudadas até o momento, são tipicamente endobranchous, ou seja, a incubação ocorre nas demibrânquias internas. No entanto, existem registros ocasionais de presença de larvas nas demibrânquias externas para um espécime de P. anomioides (Bogan \& Bouchet 1998), e também para um espécime de C. matannensis com registro de muitas larvas na demibrânquia externa (Korniushin \& Glaubrecht 2003).

O presente trabalho apresenta aspectos comparativos levando em consideração o manto, sifões, massa visceral e brânquias das três espécies de Corbicula registradas para o lago Guaíba no RS.

\section{Material e Métodos}

\section{1. Área de estudo}

O lago Guaíba localiza-se na margem oeste de Porto Alegre, compreende uma superfície aproximada de $500 \mathrm{~km}^{2}$, com cerca de $50 \mathrm{~km}$ de comprimento por $12 \mathrm{~km}$ de largura média, com uma profundidade média de $2 \mathrm{~m}$, podendo atingir 60 m na Ponta de Itapuã (Vieira \& Rangel 1988). O Parque Estadual de Itapuã está localizado ao sul do Distrito de Itapuã, no município de Viamão, entre as coordenadas $30^{\circ} 20^{\prime}$ e $30^{\circ} 27^{\prime}$ S e $50^{\circ} 50^{\prime}$ e $51^{\circ} 05^{\prime} \mathrm{W}$, a 57 km de Porto Alegre (POA). 
A área do Parque é de 5.566,50 ha e tem como limites ao sul e ao leste a laguna dos Patos e a oeste o lago Guaíba (Figura 1) (Rio Grande do Sul 1997). A praia da Pedreira (Figura 1), área do presente trabalho, uma das duas praias abertas recentemente ao público, fica às margens do Guaíba, tendo uma extensão de aproximadamente 700 m em direção ao farol de Itapuã e largura aproximada de 250 m (Rio Grande do Sul 1997). As coletas foram realizadas a partir do trapiche localizado nesta praia.

\section{Metodologia}

Foram realizadas amostragens mensais, aleatoriamente, de junho de 2002 a junho de 2003, na praia da Pedreira. Todos os exemplares vivos coletados durante aquele período foram fixados diretamente em Dubosq-Brasil, segundo Brandolini \& Amato (2001), e parte em etanol 70 GL. Foram examinados 30 exemplares, dos 344 de $C$. fluminea; 25 dos 63 de C. aff. fluminalis; e 15 dos 21 de C. largillierti. Para exame das demibrânquias e sifões os espécimes, retirados da concha, tiveram as demibrânquias internas e externas e os sifões extraídos. As demibrânquias foram observadas ao estereomicroscópio, por transparência, para visualização da formação de marsúpio e incubação nos casos de indivíduos grávidos. Os sifões foram observados ao esteriomicroscópio, quanto à forma, cor e arranjo dos tentáculos. As espécies foram identificadas conforme Martins (2004) e Martins et al. (2004).

Para a caracterização quanto à incubação das larvas adaptou-se a terminologia proposta por Hoeh et al. (Korniushin \& Glaubrecht 2003) quando definem que espécimes com incubação apenas nas demibrânquias externas são denominados ectobranchous (ectobranquiais), com incubação exclusivamente nas demibrânquias internas endobranchous (endobranquiais), e nas quatro demibrânquias tetragenous (tetrabranquiais).

\section{Resultados}

A análise morfológica comparativa das partes moles de espécimes de $C$. fluminea (Figura 2), C. aff. fluminalis (Figura 3) e C. largillierti (Figura 4) mostram as seguintes peculiaridades.

Manto: as bordas do manto das três espécies apresentam papilas no lobo mediano que se distribuem desde a região posterior até quase a região anterior, diminuindo de tamanho à medida que se aproximam da região anterior, não tendo sido observadas diferenças quanto à organização das mesmas. O manto de C. fluminea apresenta uma coloração branca, de aspecto leitoso o que contrasta com o manto branco, translúcido das outras duas espécies.

Sifões: em relação aos sifões, por se tratar de material fixado não foi possível um detalhamento maior. No entanto, foi possível constatar algumas diferenças, principalmente, em relação aos sifões inalantes e respectiva pigmentação
(Figuras 5-11). Em C. fluminea existe um denso anel de pigmentos (Figuras 5-6) ao redor dos sifões inalante e exalante, enquanto em $C$. largillierti o anel externo de pigmentos está praticamente restrito à região do sifão exalante, havendo uma concentração maior na região do diafragma que divide os sifões (Figura 10). Os espécimes de C. aff. fluminalis não apresentam anel externo de pigmentos ao redor dos sifões (Figura 8), porém, assim como nas outras duas espécies, apresentam um anel interno de pigmentos localizado junto à base dos tentáculos do sifão inalante. Cabe ressaltar que em $C$. fluminea esta pigmentação é mais intensa.

Espécimes de C. fluminea, além de apresentarem uma pigmentação mais conspícua, mostram o sifão inalante com uma organização mais simples dos tentáculos, isto é, dispostos em duas fileiras (Figura 7), uma de tentáculos filtradores e a outra de tentáculos sensoriais, conforme Britton \& Morton (1982) e Mansur \& Meier-Brook (2000). Em espécimes de C. aff. fluminalis, os tentáculos estão organizados em três ou quatro fileiras, sendo uma fileira externa de tentáculos menores e as fileiras mais internas com tentáculos maiores, aparentemente, entremeados por tentáculos de tamanhos intermediários (Figura 9). Em C. largillierti , os tentáculos no sifão inalante apresentaram uma organização, aparente, em três fileiras desencontradas, sendo a mais externa também de tentáculos menores (Figura 11), assim como observado nas outras duas espécies.

Massa visceral: a epiderme da massa visceral em espécimes de $C$. fluminea pode apresentar-se algo translúcida sendo possível visualizar, por transparência, as gônadas que ocupam grande parte desta. Em $C$. largillierti e $C$. aff. fluminalis a epiderme é bastante translúcida e fina, permitindo igualmente visualizar as gônadas que também ocupam grande parte da massa visceral. As gônadas em C. fluminea apresentam uma coloração verde acizentada e, segundo Fox (1999), correspondem aos ovários formados por folículos arborescentes, às vezes parte das gônadas apresenta-se de cor branca brilhante que, segundo o mesmo autor, correspondem aos testículos. Em espécimes de $C$. largillierti e de $C$. aff. fluminalis a coloração observada foi o branco brilhante, não tendo sido constatadas alterações na coloração e aparência das gônadas dos espécimes examinados. A glândula digestiva principalmente em espécimes de $C$. largillierti e $C$. aff. fluminalis é visível por transparência sendo bastante desenvolvida e de cor amarela. Em espécimes de C. fluminea a glândula digestiva também apresenta coloração amarela, porém dificilmente é observada por transparência por estar envolvida pelas gônadas.

Brânquias: as brânquias de C. fluminea (Figura 12) e C. aff. fluminalis (Figura 14) são bastante semelhantes diferindo da de C. largillierti (Figura 16), principalmente, quanto à demibrânquia externa, que nesta espécie apresenta um tamanho relativamente pequeno não alcançando a 
metade da altura da demibrânquia interna (Figura 17). Em C. fluminea e $C$. aff. fluminalis a demibrânquia externa atinge a metade da altura da interna (Figuras 12 e 14).

Em relação à incubação intrabranquial foram registrados espécimes grávidos para as três espécies, porém apresentando diferenças em relação ao processo de incubação. A maioria dos 20 espécimes grávidos (80\%) de C. fluminea mostrou-se endobranquial (Tabela 1, Figura 12), porém dentre estes quatro (20\%) caracterizaram-se como tetrabranquiais (Tabela 1, Figura 13). As larvas estavam densamente espalhadas por toda extensão das demibrânquias internas, já nas demibrânquias externas as larvas ficaram restritas a alguns tubos aqüíferos, em pequena quantidade em comparação as demibrânquias internas ou como o registrado para $C$. aff. fluminalis.

Dos nove spécimes grávidos de $C$. aff. fluminalis, 88,8\% apresentaram incubação tetrabranquial, com exceção de um único espécime com larvas apenas nas demibrânquias internas (Tabela 1). A quantidade de larvas presentes nas demibrânquias externas dos espécimes de $C$. aff. fluminalis (Figura 15) foi bastante grande e geralmente encontravamse amplamente espalhadas por toda sua extensão, o que também foi constatado nas demibrânquias internas.

Espécimes de C. largillierti apresentaram incubação exclusivamente endobranquial (100\%), com as larvas ocupando toda extensão das demibrânquias (Tabela 1, Figura 16). As larvas véliger observadas nas demibrânquias dos espécimes das três espécies apresentavam a forma D.

\section{Discussão}

Apesar dos inúmeros trabalhos realizados enfocando aspectos anatômicos de C. fluminea (Britton \& Morton 1979, 1982, Araújo et al. 1993, Mansur \& Meier-Brook 2000) sua comparação com outras espécies é bastante restrita, principalmente no que se refere à morfologia das partes moles. Britton \& Morton (1979) comparam pela primeira vez as partes moles de C. fluminea e C. fluminalis. Distinguiram $C$. fluminea pelos seguintes aspectos: (1), presença de uma banda de pigmentos nos tentáculos do sifão inalante; (2), a presença de um anel de pigmento internamente no sifão exalante; (3), o sifão exalante mais intensamente pigmentado externamente; (4), o número e tamanho relativo dos tentáculos sensoriais ao redor do sifão exalante menor e (5) as papilas, localizadas na região dorsal e ventral dos sifões, onde os lobos do manto se fusionam, formam uma única fileira de papilas alternadas. Entretanto, não mencionaram diferenças quanto às feições do sifão inalante, sendo este um importante caráter para identificação de espécies de Corbicula, conforme indica o estudo de Harada \& Nishino (1995) que descreveram diferenças nos sifões inalantes de espécies de Corbicula japonesas. No presente estudo constatou-se que a morfologia do sifão inalante pode ser considerada como um bom caráter para diferenciação das espécies de Corbicula aqui estudadas - C. fluminea com anel externo densamente pigmentado, abrangendo sifão inalante e exalante, tendo o sifão inalante duas fileiras de tentáculos; C. aff. fluminalis sem anel externo de pigmento, o sifão inalante com três ou quatro fileiras de tentáculos, sendo a fileira externa de tentáculos menores; C. largillierti com anel externo de pigmento restrito ao sifão exalante e diafragma, o sifão inalante com três fileiras de tentáculos, sendo a fileira externa de tentáculos menores. Aspectos da morfologia das partes moles de C. largillierti ainda não haviam sido contemplados na literatura em comparação com outras espécies.

Os sifões de Corbicula são do tipo B (Yonge 1982). Britton \& Morton (1979) e Harada \& Nishino (1995) apresentaram diagramas de sifões inalantes de espécies de Corbicula, sendo que o arranjo e forma das papilas dos espécimes do lago Guaíba mostraram-se bastante similares aos diagramas daqueles autores. Cabe ressaltar que os espécimes, do lago Guaíba, identificados como C. fluminea mostraram uma forte pigmentação externa ao redor dos sifões e seus tentáculos são mais pigmentados do que os das outras duas espécies. Além disso, os tentáculos de C. fluminea mostraram uma organização bastante simples, dispostos em duas fileiras, concordando como o observado também por Britton \& Morton (1979) e Mansur \& Meier-Brook (2000). Em espécimes identificados como $C$. aff. fluminalis observou-se a ausência de pigmentação externa ao redor dos sifões e os tentáculos pouco pigmentados internamente. Mesmo apresentando algumas diferenças, as feições apresentadas pelos espécimes de $C$. fluminea e $C$. aff. fluminalis são bastante similares ao observado por Britton \& Morton (1979), especialmente, quanto à ausência de pigmento ao redor dos sifões, o que também é mencionado por Mouthon (1981) para uma população de Corbicula do rio Tejo.

Os espécimes de $C$. largillierti apresentaram os sifões bastante semelhantes ao observado para C. fluminea, porém o anel de pigmento estava restrito ao sifão exalante, sendo a pigmentação mais concentrada na região do diafragma; diferente ao observado para $C$. fluminea, sendo que a organização dos tentáculos no sifão inalante mostrouse mais complexa.

As diferenças observadas em relação aos sifões, principalmente nos inalantes, podem representar preferências alimentares distintas entre as espécies, tal como observado por Rajagopal et al. (2000) para espécimes de $C$. fluminea e $C$. fluminalis no rio Reno, o que pode possibilitar a coexistência destas espécies.

Assim como constatado por Araújo et al. (1993), também não foram observadas diferenças capazes de discriminar as espécies de Corbicula do lago Guaíba em relação ao número e disposição das papilas do manto. No entanto, observaram-se diferenças quanto à coloração do manto que distinguiu, principalmente, C. fluminea das outras duas espécies. 
Em termos gerais, os membros do gênero Corbicula têm a reprodução e desenvolvimento heterogêneos (Park \& Kim 2003). Esta grande diversidade de padrões reprodutivos entre Corbiculidae, e especialmente em Corbicula, pode estar relacionada com o amplo espectro ecológico da família (Korniushin \& Glaubrecht 2003), principalmente se comparada com Unionoidea e Sphaeriidae que incluem somente formas de água doce e têm uma diversidade reprodutiva mais restrita do que a dos corbiculídeos (Byrne 1998, Mansur \& MeierBrook 2000, Korniushin \& Glaubrecht 2002).

Apesar dos inúmeros trabalhos que relatam aspectos reprodutivos de C. fluminea (Britton \& Morton 1982, Kraemer \& Galloway 1986, Araújo et al. 1993) são poucos os estudos desenvolvidos na América do Sul, principalmente no Brasil, referente a aspectos reprodutivos deste bivalve (Mansur et al. 2004). Schirmer (1996), para a lagoa Suzana, Planície Costeira do RS, relata a presença de espécimes de Corbicula incubando larvas nas demibrânquias internas.

No presente trabalho faz-se o primeiro registro de incubação de embriões nas demibrânquias externas de $C$. fluminea na América do Sul. Morton (1977a) também registrou a presença ocasional de larvas nas demibrânquias externas de espécimes de Hong Kong sugerindo que elas não seriam providas de nutrientes, mas provavelmente reabsorvidas por mecanismos de defesa materna.

A presença de larvas nas demibrânquias externa registrada para $C$. fluminea, assim como registrado para $P$. anomioides (Bogan \& Bouchet 1998) e para C. matannensis (Korniushin \& Glaubrecht 2003), pode ser ocasional. Tal hipótese pode ser justificada em função do pequeno número de espécimes com embriões nas demibrânquias externas (Tabela 1), associado a pouca quantidade de larvas presentes, principalmente se comparado com o encontrado em espécies tipicamente tetrabranquiais como C. possoensis (Korniushin \& Glaubrecht 2003) ou inclusive ao observado para espécimes do lago Guaíba identificados como $C$. aff. fluminalis.

A constatação de incubação por parte dos indivíduos identificados como $C$. aff. fluminalis pode gerar dúvidas quanto à identificação específica, uma vez que Britton \& Morton (1979), Morton (1986) e Araújo et al. (1993) discriminam C. fluminea de C. fluminalis pelo fato desta última não incubar suas larvas nas brânquias, sendo considerada como uma das principais características diferenciais. No entanto, Morton (1982) relata a presença de glândulas de muco nas brânquias de espécimes identificados como C. cf. fluminalis semelhantes às encontradas em C. fluminea (Morton 1977b, 1985). Byrne et al. (2000) e Korniushin \& Glaubrecht (2003) também relatam a presença destas glândulas para $C$. australis e $C$. possoensis, respectivamente. A presença destas estruturas na espécie não incubadora $C$. cf. fluminalis sensu Morton (1982) é interpretada como uma "pré-adaptação" ao desenvolvimento de incubação (Morton 1982, 1985, Korniushin \& Glaubrecht 2003).
Estudos referentes à reprodução de C. fluminalis são bastante escassos, principalmente se comparados com $C$. fluminea. O único estudo realizado fora de seu ambiente de origem é o de Rajagopal et al. (2000) que atribuem a coexistência de $C$. fluminalis e $C$. fluminea no rio Reno a diferentes períodos reprodutivos e estratégias alimentares. Portanto, faz-se necessário uma investigação sobre a biologia reprodutiva de $C$. aff. fluminalis, principalmente visando estimar a porcentagem de indivíduos hermafroditas nas populações residentes no lago Guaíba, uma vez que a incubação em corbiculídeos está relacionada aos táxons de água doce, geralmente hermafroditas (Morton 1986, Korniushin \& Glaubrecht 2003).

Ituarte (1984a) ao descrever a biologia reprodutiva de C. largillierti (Philippi, 1844) assinala que esta espécie apresenta um ciclo reprodutivo contínuo, tendo dois picos reprodutivos, um na primavera e outro no verão. E atribui este tipo de comportamento a pouca variação térmica em regiões neotropicais.

Levando em consideração as diferenças apresentadas por Britton \& Morton (1979, 1982, 1986) e Araújo et al. (1993) quanto às partes moles de $C$. fluminea e C. fluminalis e levando-se, ainda, em consideração as diferenças populacionais também registradas por estes autores, bem como as diferenças no comportamento reprodutivo, podese sugerir que realmente uma terceira espécie de Corbicula, juntamente com C. fluminea e C. largillierti, está presente no lago Guaíba sendo identificada, provisoriamente, como C. aff. fluminalis.

Como aspecto a ser levado em consideração, merece ser ressaltado que as características aqui apresentadas para três populações de Corbicula encontradas em simpatria no lago Guaíba, em mesmo período de amostragem, convivem em mesmas condições, isto é, de profundidade, luminosidade, disponibilidade de alimento, entre outros.

\section{Agradecimentos}

À Dra. Maria Cristina D. Mansur e Dra. Cláudia T. Callil pelo apoio e bibliografia; ao Dr. Cristián F. Ituarte pela bibliografia; Daniel Pimpão pelo auxílio nas coletas, criticas e sugestões; Capes, pela bolsa concedida ao autor sênior.

\section{Referências Bibliográficas}

ARAÚJO, R., MORENO, D. \& RAMOS, A. 1993. The Asiatic clam Corbicula fluminea (Müller, 1774) (Bivalvia: Corbiculidae) in Europe. Am. Malacol. Bull. 10 (1): 39-43.

BOGAN, A. \& BOUCHET. P. 1998. Cementation in freshwater bivalve family Corbiculidae (Mollusca: Bivalvia): a new genus and species from Lake Posso, Indonesia. Hydrobiologia 389: 131-139. 
BRANDOLINI, S.V.P.B. \& AMATO, S.B. 2001. Desenvolvimento de Eurytrema coelomaticum (Giard \& Billet) (Digenea, Dicrocoeliidae) em Bradybaena similiaris (Férussac) (Gastropoda, Xanthonychidae). Rev. Bras. Zool. 18(2): 499-510.

BRITTON, J.C. \& MORTON, B. 1979. Corbicula in North America: the evidence reviewed and evalluated. In Proceedings of the First International Corbicula Symposium. Texas Christian University Reserarch Foudation , Texas, p. 249-287.

BRITTON, J.C. \& MORTON, B. 1982. A dissection guide, field and laboratory manual for the introduced bivalve Corbicula fluminea. Malacol. Rev. Suppl. 3: 1-82.

BRITTON, J.C. \& MORTON, B. 1986. Polumorphism in Corbicula fluminea (Bivalvia: Corbiculoidea) from North America. Malacol. Rev. 19: 1-43.

BYRNE, M. 1998. Reproduction of river and lake populations of Hyridella depressa (Unionacea: Hyriidae) in New South Wales: implications for their conservation. Hydrobiologia 389: 29-43.

BYRNE, M., PHELPS, H., CHURCH, T., ADAIR, V., SELVAKUMARASWAMY, P. \& POTTS, J. 2000. Reproduction and development of the freshwater clam Corbicula australis in southeast Australia. Hydrobiologia 418: 185-197.

COUNTS III, C.L. 1986. The zoogeography and history of the invasion of the United States by Corbicula fluminea (Bivalvia: Corbiculidae). Am. Malacol. Bull., Special Edition 2: 7-39.

FOCHT, T. \& VEITENHEIMER-MENDES, I.L. 2001. Distribuição de Neocorbicula limosa (Maton) (Bivalvia, Corbiculidae) no lago Guaíba, Rio Grande do Sul, Brasil. Rev. Bras. Zool. 18 (1): 35-43.

FOX, R. 1999. Invertebrate Anatomy Corbicula fluminea. http://www.lander.edu/rsfox/corbicula.html (último acesso 30/05/ 2002).

HARADA, E. \& NISHINO, M. 1995. Differences in inhalant siphonal papillae among the Japanese species of Corbicula (Mollusca: Bivalvia). Publ. Seto Mar. Biol. Lab. 36 (5-6): 389-408.

ITUARTE, C.F. 1984a. Aspectos biológicos de las poblaciones de Corbicula largillierti Philippi (Mollusca Pellecypoda) em el Rio de La Plata. Rev. Mus. La Plata Secc. Zool. 13 (143): 231-247.

ITUARTE, C.F. 1984b. El fenomeno de incubacion branquial en Neocorbicula limosa (Maton), 1809 (Mollusca Pelecypoda). Neotropica 30 (83): 43-54.

ITUARTE, C.F. 1994. Corbicula and Neocorbicula (Bivalvia: Corbiculidae) in the Paraná, Uruguay, and Río de la Plata basins. Nautilus 107 (4): 129-135.
KORNIUSHIN, A.V. \& GLAUBRECHT, M. 2002. Phylogenetic analysis based on the morphology of viviparous freshwater clams of the family Sphaeriidae (Mollusca, Bivalvia, Veneroida). Zool. Scr. 31 (5): 415-459.

KORNIUSHIN,A.V. \& GLAUBRECHT, M. 2003. Novel reproductive modes in freshwater clams: brooding and larval morphology in Southeast Asian taxa of Corbicula (Mollusca, Bivalvia, Corbiculidae). Acta Zoologica 84: 293-315.

KRAEMER, L.R. \& GALLOWAY, M.L. 1986. Larval development of Corbicula fluminea (Müller) (Bivalvia: Corbiculacea): an appraisal of its heterochrony. Am. Malacol. Bull., Special Edition 2: 61-79.

MACKIE, G.L. 1986. Adaptations of Pisidiidae (Heterodonta: Corbiculacea) to freshwater habitats. Am. Malacol. Bull., Special Edition 2: 113-124.

MANSUR, M.C.D., CALLIL, C.T., CARDOSO, F.R., SANTOS, C.P. \& IBARRA, J.A.A. 2004. Uma retrospectiva e mapeamento da invasão de espécies de Corbicula (Mollusca, Bivalvia, Veneroida, Corbiculidae) oriundas do sudeste asiático, na América do Sul. In Água de lastro e Bioinvasão (J.S.V. Silva \& R.C.C.L. Souza eds.). Interciências, Rio de Janeiro, p. 39-58

MANSUR, M.C.D. \& MEIER-BROOK, C. 2000. Morphology of Eupera Bourguignat 1854, and Byssanodonta Orbigny 1846 with contributions to the phylogenetic systematics of Sphaeriidae and Corbiculidae (Bivalvia: Veneroida).Arch. Molluskenkd. 128 (1/2): 1-59.

MARTINS, D.S. 2004. Aspectos comparativos da morfologia e comportamento reprodutivo de três espécies de Corbicula Mühlfeld, no lago Guaíba, Rio Grande do Sul, Brasil (Bivalvia, Corbiculidae). Porto Alegre. Dissertação de Mestrado, Universidade Federal do Rio Grande do Sul, Porto Alegre.

MARTINS,D.S.,VEITENHEIMER-MENDES,I.L \& FACCIONI-HEUSER,M.C. 2004. Corbicula (Bivalvia, Corbiculidae) em simpatria no lago Guaíba, Rio Grande do Sul, Brasil. Biociências 12(2):129-138.

MCMAHON, R.F. 1983. Ecology of an invasive pest bivalve, Corbicula. In The Mollusca (W.D. Russel-Hunter ed.). Academic Press, New York, vol.6, p. 505-561.

MORTON, B. 1977a. The occurrence of inflamatory granulomas in the ctenidial marsupium of Corbicula fluminea (Mollusca: Bivalvia): a consequence of larval incubation. J. invertebr. Pathol. 30: 5-14.

MORTON, B. 1977b. The population dynamics of Corbicula fluminea (Bivalvia: Corbiculacea) in Plover Cove Reservoir, Hong Kong. J. Zool. 181: 21-42.

MORTON, B. 1982. Some aspects of the population structure and sexual strategy of Corbicula cf. fluminalis (Bivalvia: Corbiculacea) from the Pearl River, people's Republic of China. J. Mollusca Stud. 48: 1-23. 
MORTON, B. 1985. The reproductive strategy of the mangrove bivalve Polymesoda (Geloina) erosa (Bivalvia: Corbiculoidea) in Hong Kong. Malacol. Rev. 18: 83-89.

MORTON, B. 1986. Corbicula in Asia - an updated synthesis. Am. Malacol. Bull., Special Edition 2: 113-124.

MORTON, B. 1991. Do the Bivalvia demonstrate environment-specifc sexual strategies? A Hong Kong model. J. Zool. 223: 131-142.

MOUTHON, J. 1981. Sur la présence en France et au Portugal de Corbicula (Bivalvia: Corbiculidae) originaire d'Asie. Basteria 45: 109-116.

PARK, J-K. \& Ó FOIGHIL, D. 2000. Sphaeriid and Corbiculid clams represent separate heterodont bivalve radiations into freshwater environments. Mol. Phylogenet. Evol. 14(1): 75-88.

PARK, J-K. \& KIM, W. 2003. Two Corbicula (Corbiculidae: Bivalvia) mitochondrial linages are widely distributed in Asian freshwater environment. Mol. Phylogenet. Evol. 29:529-539.

PARODIZ, J.J. \& HENNINGS, L. 1965. The Neocorbicula (Mollusca, Pelecypoda) of the Parana-Uruguay basin, South America. Ann. Carnegie Mus. 38 (3): 69-95.

RAJAGOPAL, S., VAN DER VELDE, G. \& BIJ DE VAATE, A. 2000. Reproductive biology of the Asiatic clams Corbicula fluminalis and Corbicula fluminea in the river Rhine. Arch. Hydrobiol. 149 (3): 403-420.

RIO GRANDE DO SUL 1997. Plano de Manejo: Parque Estadual de Itapuã. Secretaria da Agricultura e Abastecimento, Departamento de Recursos Naturais Renováveis, Porto Alegre, p. 1- 157.

SCHIRMER, C.L., 1996. Aspectos da biologia de Corbicula fluminea e Neocorbicula limosa (Bivalvia: Corbiculidae) na lagoa da Suzana, Rio Grande do Sul, Brasil. Dissertação de Mestrado, Universidade Federal do Rio Grande do Sul, Porto Alegre.

VIEIRA, E.F. \& RANGEL, S.R.S. 1988. Planície Costeira do Rio Grande do Sul: geografia física, vegetação e dinâmica sócio-demográfica. Sagra, Porto Alegre.

YONGE, C.M. 1982. Mantle margins with a revision of siphonal types in the Bivalvia. J. Mollusca Stud. 48: 102-103.
Título: Aspectos morfológicos e incubação em três espécies de Corbicula Mühlfeld, no lago Guaíba, Rio Grande do Sul, Brasil (Bivalvia, Corbiculidae).

Autores: Martins, D.S.; Veitenheimer-Mendes, I.L.; Faccioni-Heuser, M.C.

Biota Neotropica, Vol. 6 ( número 2 ): 2006

http://www.biotaneotropica.org.br/v6n2/pt/ abstract?article+bn02806022006

Recebido em 15/09/05 -Versão reformulada recebida em 18/04/06 - Publicado 07/06/06

ISSN 1676-0603 
Tabela 1. Resultados do exame das demibrânquias de espécimes de três táxons de Corbicula amostrados no lago Guaíba, RS, entre junho/2003 e junho/2004.

Table 1. Results of examination of demibranchs from three specimens of Corbicula collected in the Guaiba Lake, RS, between June 2003 and June 2004.

\begin{tabular}{|c|c|c|c|c|c|}
\hline Táxon & $\begin{array}{l}\mathrm{N}^{\circ} \text { espécimes } \\
\text { examinados }\end{array}$ & $\begin{array}{c}\mathrm{N}^{\circ} \text { espécimes } \\
\text { grávidos } \\
(\%)\end{array}$ & $\begin{array}{c}\mathrm{N}^{\circ} \text { espécimes } \\
\text { grávidos } \\
\text { tetrabranquiais } \\
(\%)\end{array}$ & $\begin{array}{l}\text { Menor tamanho de } \\
\text { espécime grávido } \\
(\mathrm{mm})\end{array}$ & $\begin{array}{c}\text { Variação do } \\
\text { comprimento dos } \\
\text { espécimes } \\
\text { examinados }(\mathrm{mm})\end{array}$ \\
\hline C. fluminea (Müller) & 30 & $20(66,6 \%)$ & $4(20 \%)$ & 36,58 & $23,72-45,46$ \\
\hline C. aff. fluminalis (Müller) & 25 & $9(36 \%)$ & $8(88,8 \%)$ & 23,36 & $23,36-29,78$ \\
\hline C. largillierti (Philippi) & 15 & $3(20 \%)$ & $0(0 \%)$ & 26,58 & $23,22-28,70$ \\
\hline
\end{tabular}




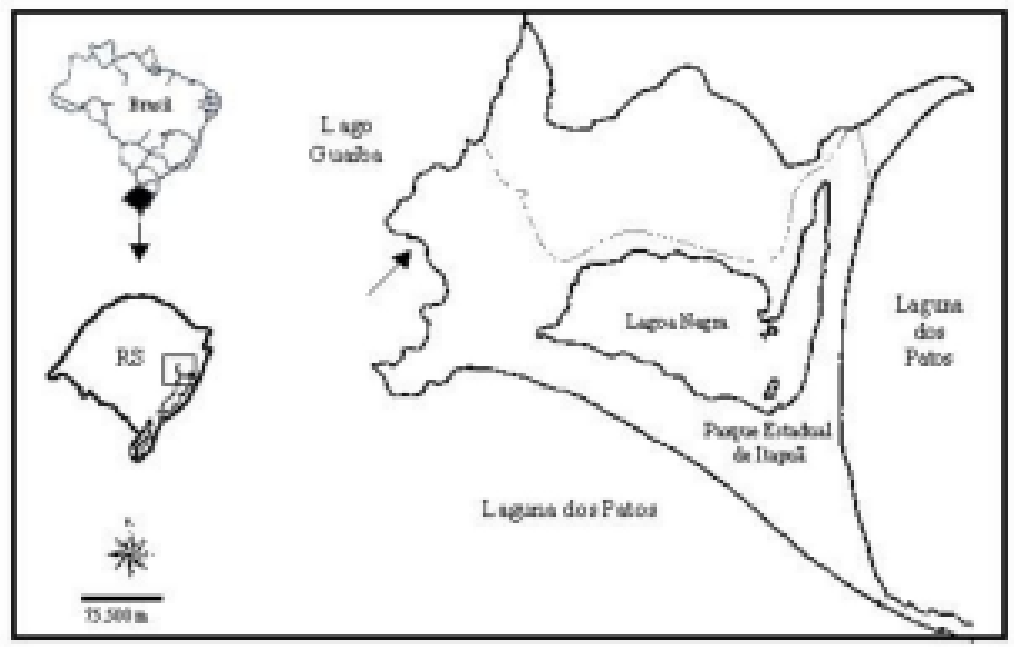

Figura. 1. Localização da Localização da Praias da Pedreira (个), Parque Estadual de Itapuã, Viamão, RS, às margens do lago Guaíba. Figure 1. Location of Pedreira Beach (个), Parque Estadual de Itapuã, Viamão, RS, at the edge of Guaíba Lake. 

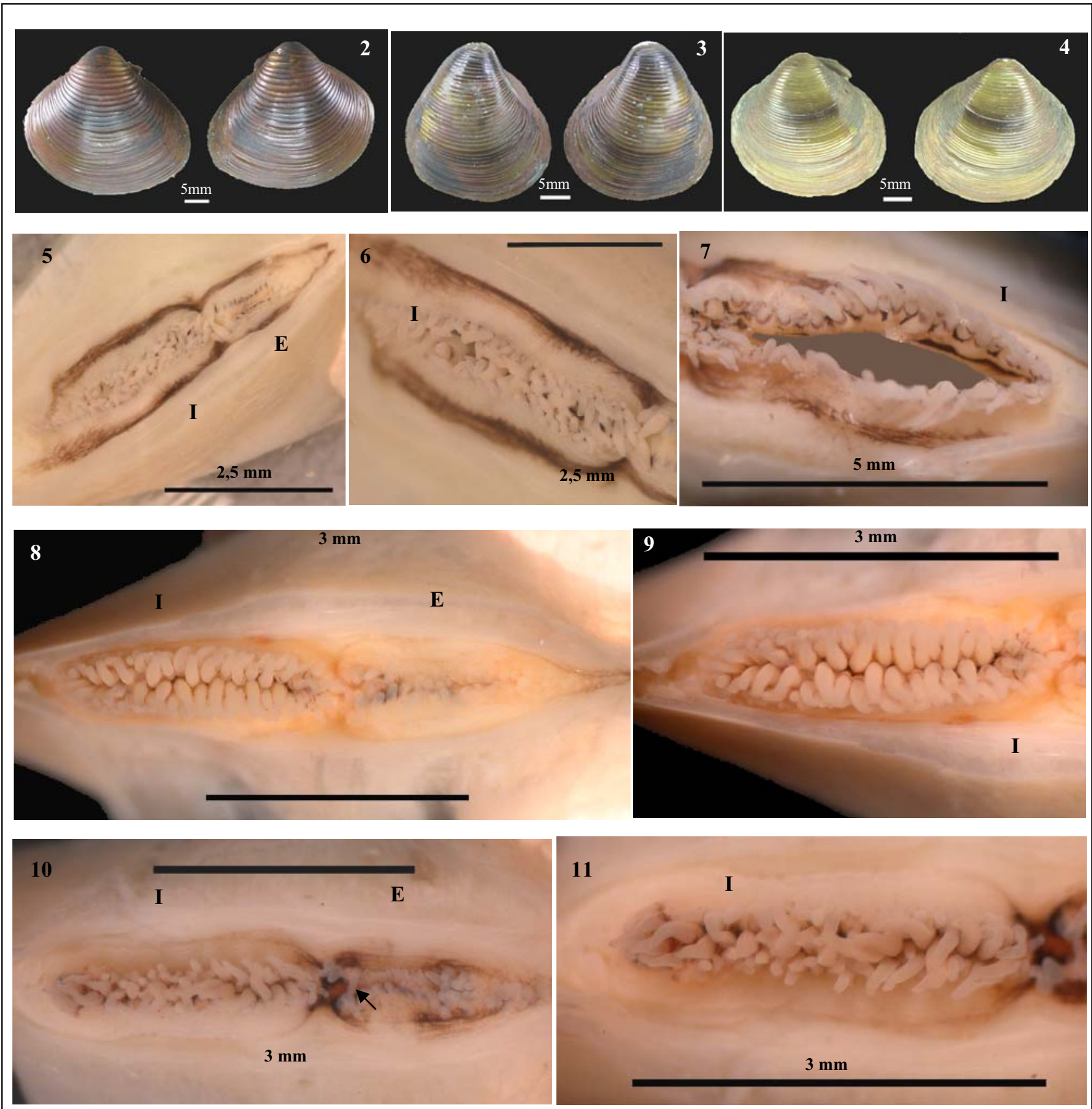

Figuras 2-11. (2-4) Conchas (2) Corbicula fluminea (Müller, 1774); (3) Corbicula aff. fluminalis (Müller, 1774); (4) Corbicula largillierti (Philippi, 1844); (5-7) Corbicula fluminea: (5) vista geral dos sifões; (6) vista do sifão inalante destacando a densa pigmentação ao redor; (7) vista do sifão inalante destacando o arranjo dos tentáculos; (8-9) Corbicula aff. fluminalis (Müller, 1774): (8) vista geral dos sifões; (9) vista do sifão inalante; (10-11) Corbicula largillierti (Philippi, 1844): (10) vista geral dos sifões, destacando presença de pigmento (-); (11) vista do sifão inalante. (I) Sifão inalante; (E) sifão exalante.

Figures 2-11. (2-4) Shells (2) Corbicula fluminea (Müller, 1774); (3) Corbicula aff. fluminalis (Müller, 1774); (4) Corbicula largillierti (Philippi, 1844); (5-7) Corbicula fluminea: (5) general view of the siphons; (6) view of the inhalant siphon showing the dense pigmentation around; (7) ) view of the inhalant siphon showing the tentacles arrangement; (8-9) Corbicula aff. fluminalis (Müller, 1774): (8) general view of the siphons; (9) inhalant siphon; (10-11) Corbicula largillierti (Philippi, 1844): (10) siphons general view, showing the pigmentation (-); (11) Inhalant siphon. (I) Inhalant siphon; (E) exhalant siphon. 

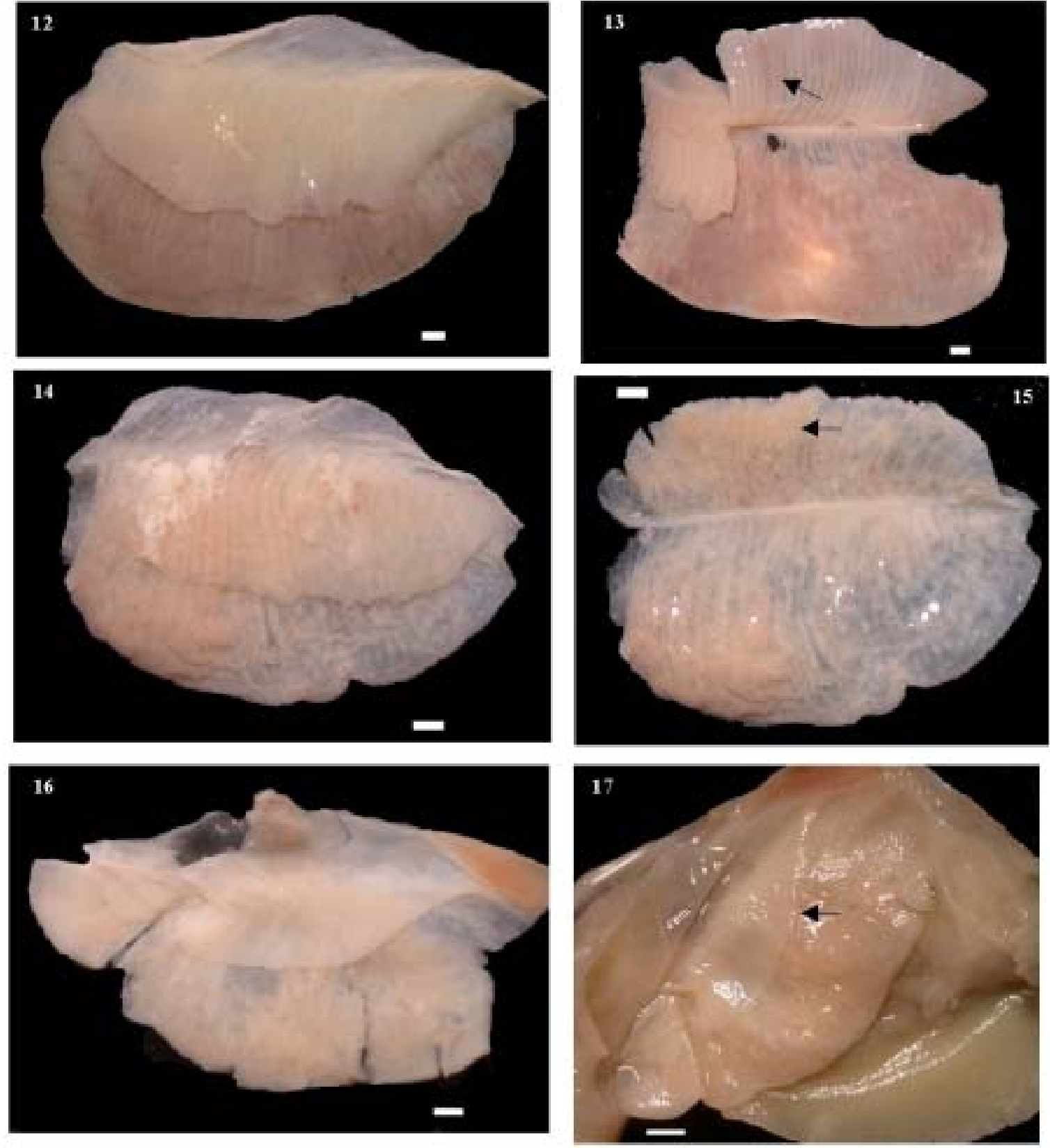

Figuras 12-17. (12-13) Corbicula fluminea (Müller, 1774), vista geral das demibrânquias: (12) com larvas na demibrânquia interna; (13) com larvas nas demibrânquias externas ( $\uparrow$ ) e internas; (14-15) Corbicula aff. fluminalis (Müller, 1774), vista geral das demibrânquias: (14), com larvas nas demibrânquias externas e internas; (15) destacando a grande quantidade de larvas na demibrânquia externa (-); (16-17) Corbicula largillierti (Philippi, 1844), vista geral das demibrânquias: (16), com larvas na demibrânquia interna; (17) destacando a pequena dimensão da demibrânquia externa (个). Escalas: figuras (12-16): $1 \mathrm{~mm}$; figura (17) $3 \mathrm{~mm}$.

Figures 12-17. (12-13) Corbicula fluminea (Müller, 1774), general view of the demibranchs: (12) with larvae in the inner demibranch; (13) with larvae in the outer ( $\uparrow)$ and inner demibranchs; (14-15) Corbicula aff. fluminalis (Müller, 1774), general view of the demibranchs: (14) with larvae in the outer and inner demibranchs; (15) outstanding the large number of larvae in the outer demibranch (-); (16-17) Corbicula largillierti (Philippi, 1844), general view of the demibranchs: (16), ) with larvae in the inner demibranch; (17) outstanding the small size of the outer demibranch $(\uparrow)$. Scales: figures (12-16): $1 \mathrm{~mm}$; figure (17) $3 \mathrm{~mm}$. 